Veritas E Scientia

Vol. 8. № 1.

Enero - Junio del 2019.

ISSN Edición Online: 2617-0639

https://doi.org/10.47796/ves.v8i1.105

\title{
FACTORES PRINCIPALES DE INEFICACIA DE LA LEY DE RELACIONES COLECTIVAS DE TRABAJO EN LA REGIÓN TACNA 2001-2015
}

\section{MAIN FACTORS OF INEFFICIENCY OF THE LAW OF COLLECTIVE LABOR RELATIONS IN THE TACNA REGION 2001-2015}

\author{
Mario Gordillo Cossio ${ }^{1}$ \\ Elva Acevedo Velásquez ${ }^{2}$ \\ Presentado: 11/08/2018 \\ Aceptado: $17 / 11 / 2019$ \\ Publicado online:17/07/2019
}

\begin{abstract}
RESUMEN
El estudio ha permitido relacionar las variables dependiente $e$ independiente en el contexto social de un periodo de tiempo extenso para determinar su comportamiento y correlación, planteándose el objetivo de determinar el grado de inflexión entre ambas variables que afecte significativamente el clima laboral colectivo en la ciudad de Tacna; así como de obtener información oficial y analizar comparativamente los efectos y características que plantea el cumplimiento de la normatividad vigente en materia de relaciones colectivas de trabajo en la región de Tacna. Se ha utilizado el método científico y dentro de él el método inductivo-deductivo. El tipo de investigación es cualitativo y el nivel explicativo de corte transversal. En cuanto al diseño es no experimental obteniéndose la información de la observación directa. La población del estudio es la referida a la ciudad de Tacna tomándose la muestra de las organizaciones sindicales de la región para determinar el comportamiento y grado de eficacia de la norma. Los instrumentos utilizados fueron la entrevista y cuestionario. El estudio concluye que entre las variables independientes de normatividad y relaciones colectivas débiles existe correlación con los efectos que ha tenido la crisis sindical en el Perú desde la década del noventa, ello porque las normas que regulan las Relaciones Colectivas de Trabajo no promueven el adecuado ejercicio del derecho colectivo de los trabajadores sino su debilitamiento. Se han podido establecer deficiencias normativas en cuanto al grado de cumplimiento de las recomendaciones de la Organización Internacional del Trabajo y a la ausencia de políticas de promoción de la negociación colectiva en el ámbito estatal, lo que ha generado consecuencias económico-sociales directas en la población sindicalizada y no sindicalizada. La modificación normativa en esta materia
\end{abstract}

\footnotetext{
1 Doctor en Derecho

2 Doctora en Derecho y Vicerrectora Académica de la Universidad Privada de Tacna.
} 
resulta entonces de urgente necesidad, presentando una primera propuesta.

Palabras clave: relaciones colectivas

\begin{abstract}
The study has allowed to relate the dependent and independent variables in the social context of an extended period of time to determine their behavior and correlation, raising the objective of determining the degree of inflection between both variables that significantly affect the collective working climate in the city Of Tacna; As well as of obtaining official information and analyzing comparatively the effects and characteristics of compliance with current regulations on collective labor relations in the Tacna region. The scientific method has been used it the inductive-deductive. The type of research is qualitative and the explanatory level of cross-section. As for the design is non-experimental obtaining the information of direct observation. The population of the study is that referred to the city of Tacna taking the sample of trade union organizations in the region to determine the behavior and degree of effectiveness of the standard. The instruments used were the interview and questionnaire. The study concludes that among the independent variables of normativity and weak collective relations there is correlation with the effects that the trade union crisis has had in Peru since the nineties, because the norms that regulate Collective Labor Relations do not promote the adequate exercise of the collective right of workers but their weakening. Regulatory deficiencies have been identified in terms of compliance with the recommendations of the International Labor Organization and the lack of policies to promote collective bargaining at the state level, which has led to direct social and economic consequences for the unionized population and not unionized. The normative change in this matter is there fore of urgent necessity, presenting a first proposal.
\end{abstract}

Keywords: collective relations

\title{
INTRODUCCIÓN
}

Una de las actividades más importantes luego de la supervivencia es el trabajo, y desde tiempos ancestrales el ser humano ocupaba su día en realizar estas dos actividades básicas. Con el nacimiento de la agricultura y el consecuente surgimiento de grupos sociales se hizo más fácil mantener la seguridad de los individuos. Todavía el ser humano lucha diariamente para evitar su extinción, sólo que esta vez no sólo es física, también se puede hablar de una extinción social, que eventualmente puede afectar a la persona llevándola en algunos casos a la muerte. La Constitución Política del Perú consagra el derecho al Trabajo ${ }^{1}$ como parte de los derechos fundamentales de la persona, esto es así porque es a través del trabajo que se logra no sólo asegurar la subsistencia del ser humano, también es clave en el desarrollo de su individualidad y su crecimiento personal ${ }^{2}$. La inexistencia de una ocupación laboral le recorta al individuo la posibilidad de acceder a recursos económicos para su manutención, genera el rechazo social y le da una ubicación desventajosa en la escala social del grupo al que pertenece. Dentro del amplio bagaje de normas que en materia laboral han sido desarrolladas en nuestro país, cobran especial importancia las destinadas a regular las relaciones laborales colectivas de trabajo. Se buscó analizar si las normas actuales

\footnotetext{
${ }^{1}$ Artículo 2o, numeral 15, Constitución Política del Perú: ....A trabajar libremente, con sujeción a ley”.

${ }^{2}$ Artículo 22ำ, Constitución Política del Perú: ..."Es base del bienestar social y un medio de realización de la persona".
} 
responden de manera efectiva a la necesidad nacional o cuál sería la mejor estructura normativa en el ideal de las perspectivas socio laborales; para ello debe partirse del contexto histórico nacional y contrastarse con las realidades vividas bajo la regulación de la Ley de Relaciones Colectivas de Trabajo $N^{\circ}$ 25593, su Reglamento el D.S. Nº11-92-TR y las normas que posteriormente la han modificado.

En el sistema peruano de relaciones laborales se observa una mezcla de conceptos jurídicos-normativos y actitudes o costumbres socio laborales que no sólo no guardan relación entre sí, sino que además representan un obstáculo para el desarrollo armónico de aquéllas. Con la modificación de la anterior Ley de Relaciones Colectivas de Trabajo N²5593 y su Reglamento el Decreto Supremo N011-92-TR, el Estado propendió más bien a la flexibilización de las relaciones laborales, así como de la actividad sindical expresadas como política de gobierno.

El derecho de sindicación tiene su origen en la necesidad generalizada que tuvieron las clases obreras, en los albores de la República. El primer registro que se tiene de una paralización de actividades que puede llamarse huelga, con consecuencias de inmediata repercusión social, nos remonta hasta la época del Virreinato, época no precisamente notable por el respeto a los derechos humanos. Eventualmente, nuevos movimientos se sucedieron en los años posteriores, ya iniciada la República, como las huelgas de tipógrafos en 1892, de los trabajadores textiles de Vitarte en 1896 (singularmente violentos), de los portuarios en 1904, de tranviarios en 1905, luego en 1908 la paralización de los trabajadores azucareros en reclamo de la jornada de 8 horas, cuya extrema violencia en las haciendas azucareras de Casagrande, Cartavio y Chiclín originaron una fuerte represión para, finalmente en 1913, debido a la huelga de los obreros portuarios, lograr que el gobierno de Guillermo Billinghurst dicte la primera norma reguladora al respecto, el decreto supremo de 24 de Enero de 1913, aun cuando esta norma no fue de alcance nacional. Es recién con la dación del decreto supremo de 15 de enero de 1919 que se implanta la jornada máxima legal con carácter general, a resultas de la huelga promovida en el Congreso de la Federación Obrera en Lima, seguida por los obreros textiles que paralizaron la capital los días 13, 14 y 15 de enero aquel año. (Ackerman, et al., 1996).

El derecho de huelga se incluyó en la Constitución de 1979 como un derecho expresamente reconocido ${ }^{1}$ pero que; sin embargo, no tuvo durante el tiempo que dicha Carta Magna rigió, el desarrollo normativo esperado. Con la dación de la Ley de Relaciones Colectivas de Trabajo, D.L. N² 25593 en Julio de 1992, se logra un desarrollo normativo y es ratificado en la actual Constitución vigente desde 1993 que la consagra como un derecho de rango constitucional y que incluye en su artículo $28^{\circ}$ otros derechos colectivos como los de sindicación y negociación colectiva, garantizando la libertad sindical. La referida Ley como su Reglamento, el Decreto Supremo N 011-92-TR, desarrollaron ampliamente todos los aspectos de la vida sindical. Según Ley $N^{\circ} 27912$ de enero de 2003 se modificó la legislación anterior. Tal ley originó la promulgación del D.S. N 010-2003-TR que aprueba el Texto Único Ordenado de la Ley de Relaciones Colectivas de Trabajo, actualmente en vigencia.

Es necesario definir de manera más precisa cuáles son los ejes de inflexión por los cuales la normatividad no cumple actualmente en nuestro país los objetivos o principios para los cuales ha sido desarrollada. Así partimos por analizar la realidad laboral, sindical y empresarial, de manera que se determine claramente los aspectos a mejorar o desarrollar.

Umberto Romagnoli, propone desde 1992, el mejoramiento de las relaciones laborales y la protección del trabajador como mejor medio del desarrollo armónico empresarial y económico de los países, Romagnoli H. (1992). Américo Plá Rodríguez, señaló que: "en todos los países, la tendencia es a combatir el Derecho Laboral", debido a tres razones: auge del neoliberalismo, difusión del desempleo y debilitamiento del

\footnotetext{
${ }^{1}$ Artículo 55으, Constitución de 1979: ..."Las huelgas es derecho de los trabajadores. Se ejerce en la forma que establece la Ley".
} 
movimiento sindical. Sindicato Médico del Uruguay. (2000). Primer Foro sobre Desregulación del Derecho del Trabajo en el SMU. Sanguinetti (2013) efectúa un análisis sobre la 'deconstrucción' del Derecho del Trabajo de base nacional en la era de la globalización, en el cual pone de manifiesto la precaria vigencia que pueden mantener las normas laborales destinadas a proteger a los trabajadores. Perulli (como se citó en Sanguinetti, 2013) señala que se convierte en una "inédita competición entre sistemas sociales". (Neves, et al., 2010) sostiene que las organizaciones sindicales deben modificar su esencia para no quedar desfasadas.

En este nuevo panorama las normas en materia sindical también deben adecuarse; así Pasco (2010) afirma. "quien carece de un empleo, en cambio, carece de todo" Neves, et al., 2010, concluye que los sindicatos deben adecuarse a esta nueva realidad para no caer en la obsolescencia; tal cambio atañe por lógica a las normas que los regulan.

\section{OBJETIVOS}

a) Conocer el factor que incide en el debilitamiento de las organizaciones sindicales y en qué medida la regulación tuitiva y el adecuado ejercicio del derecho colectivo del trabajo de los trabajadores fomentarían la eficacia de la Ley de Relaciones Colectivas de Trabajo.

b) Conocer en qué medida la Ley de Relaciones Colectivas de Trabajo debería guardar armonía con las disposiciones constitucionales de protección al trabajo.

\section{METODOLOGÍA}

Investigación descriptiva, analítica, explicativa y correlacional. No Experimental. El estudio se centró sobre las organizaciones y conflictos sindicales que se han dado en la región Tacna, sean de trabajadores públicos o privados, los mismos que, durante el periodo de estudio han alcanzado la cantidad de 112 (ciento doce), conforme al registro oficial de la Dirección Regional de Trabajo y Promoción del Empleo de Tacna. Se seleccionó una muestra de 68 representantes de organizaciones. Se realizó un análisis bibliográfico, encuesta, entrevista y análisis documental. El trabajo de campo se realizó en dos partes, así en un primer momento fue la entrevista y la segunda parte consistió en el análisis documental.

\section{RESULTADOS}

Resulta de especial atención que durante el periodo de estudio se haya evidenciado un crecimiento de organizaciones sindicales de la actividad pública. Este crecimiento obedece tanto a la creación de nuevos sindicatos como a la división de los ya existentes y en su mayoría no responden tanto a la singularidad de su función, sino al empoderamiento que han tenido (en los últimos años) los servidores públicos sobre sus derechos laborales, utilizando este mecanismo en un efectivo ejercicio libre de su derecho de sindicación como contrapeso del poder estatal. El crecimiento de organizaciones sindicales en el sector público es inversamente proporcional al decrecimiento que han tenido los sindicatos en el sector privado. El ejercicio de la huelga es uno de los principales instrumentos de presión; sin embargo, es importante resaltar que las condiciones y circunstancias que rodean el ámbito público son muy diferentes al sector privado.

Si las normas que regulan las Relaciones Colectivas de Trabajo no tienen un carácter dinámico que responda la cambiante realidad nacional es, en buena medida, por la falta de inclusión de procesos de revisión que faciliten la adecuación a los nuevos escenarios económicos nacionales e internacionales.

La rama de construcción civil ha sido la que en los últimos años ha generado no sólo el incremento de la constitución de organizaciones sindicales, también ha originado conflictos entre sus propios agremiados porque conjuntamente con ellos se dispersaron los intereses comunes de dichos organismos. Si bien el nivel de negociación se ha mantenido en una fluctuación adecuada, en la mayoría de los casos va 
aparejado con el apoyo de la Autoridad Administrativa de Trabajo; asimismo, se observa que, a partir de la última década hay un incremento de la resolución del pliego a través del arbitraje, lo que es indicativo del fracaso de las negociaciones.

\section{Conflictos Sindicales.}

Dentro de lo regulado por la Ley de Relaciones Colectivas de Trabajo, su Reglamento, modificatorias y el Texto Único Ordenado se han previsto distintos procesos, así como los mecanismos destinados a regular los mismos; todos a cargo de la Autoridad Administrativa de Trabajo y que guardan estrecha relación con las actividades básicas de la vida sindical (creación, registro, negociación de pliego de reclamos, huelga, cancelación, licencias). En esta parte, referida a la evaluación del grado de eficacia de la norma en la disminución de la conflictividad, encontramos el primer vacío normativo.

Presentamos la figura 01 que recoge aquellas situaciones resueltas en la vía administrativa pero que no corresponden a ninguno de dichos temas:

Se aprecia una tendencia creciente de las reuniones extra proceso que ejecuta la Dirección de Prevención y Solución de Conflictos de la Dirección Regional de Trabajo y Promoción del Empleo de Tacna.

En cuanto a los procesos judiciales a cargo de la Corte Superior de Justicia de Tacna, se encuentra también una tendencia creciente. (Ver Figura 02):

Al igual que en el ámbito administrativo, los procesos judiciales también registran un alza a partir del inicio del presente siglo, en su mayoría con las organizaciones sindicales como demandantes.

Respecto a la extinción de organizaciones sindicales entre los años 2001 - 2015, es importante resaltar que sobre este tema versan dos aspectos:

a) La disolución del sindicato, que opera por acuerdo de la mayoría absoluta de sus agremiados.

b) La cancelación del registro sindical, cuya competencia estuvo a cargo de la Autoridad Administrativa de Trabajo hasta la modificación de la Ley de Relaciones Colectivas mediante la Ley № 27912 siendo, a partir de su promulgación, competencia del Juez de Trabajo. Aun así, se registraron pocas incidencias en esta materia.

El fracaso de la negociación colectiva genera un recelo en las partes por diversas causas, en principio es la evidencia objetiva de su imposibilidad de entendimiento; yendo más a fondo, con un panorama de conflicto se ponen en riesgo los intereses de cada uno de los actores involucrados y el propio bienestar social general.

En el periodo observado se aprecia un ascenso constante, aunque no abrupto de la recurrencia de huelgas; en promedio el porcentaje de ascenso es del 4.1\%; sin embargo, analizando los valores concretos correspondientes a los años 2001 y 2011, se aprecia un incremento del 100\% del total de paralizaciones registradas en ese rango de tiempo. (Figura 3). 


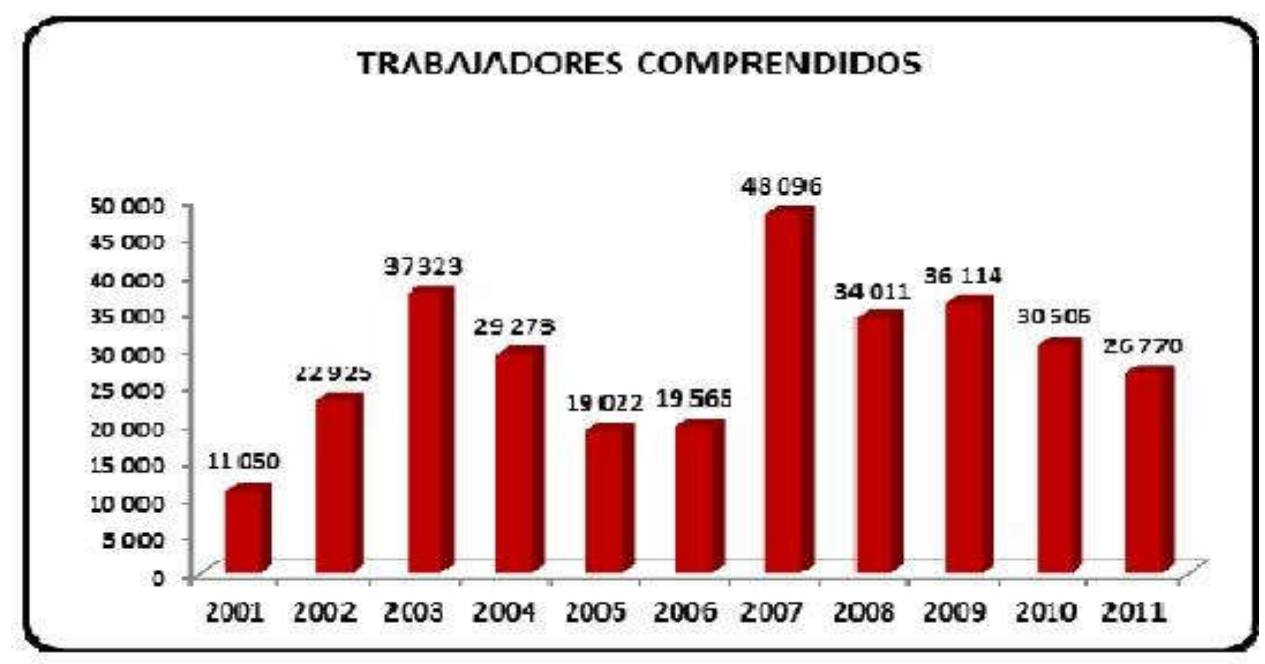

Figura 1: Trabajadores participantes en Huelgas - Perú (2001 al 2011).

Fuente: Oficina de Estadística (2011). Las Huelgas en el Perú. Ministerio de Trabajo y promoción del Empleo. Lima.

Frente a esta situación debemos considerar la importancia que reviste el empoderamiento de la conciliación como medio de solución de conflictos. Ormachea (2000) presenta la siguiente esquematización teórica de tres formas de solución de conflictos.

Por la naturaleza histórica del trabajo vemos en su desarrollo muchos elementos que no sólo no pertenecen a nuestra realidad originaria (como país), tampoco en cuanto a nuestra realidad histórica. Corresponde al Estado la responsabilidad de fomentar, promocionar, respaldar y proteger las normas que sobre relaciones colectivas se han considerado como mínimamente necesarias (OIT, 1986). En tanto ello no se dé en la realidad, la lucha de la clase trabajadora por el respeto a sus derechos fundamentales al trabajo, a la vida digna y a la salud no puede quedar al precario logro que puedan alcanzar gracias al desprendimiento forzado y aún inoportuno que pueda hacer el empleador en un momento determinado.

El Derecho Colectivo, las normas emitidas por el gobierno en materia de relaciones colectivas, sirven de marco para el correcto y eficaz ejercicio de los derechos individuales de los trabajadores. En este aspecto (Neves, et al., 1996) señala que la creación del trabajo internacional se da por "i) el desplazamiento de trabajadores de un estado a otro; y la ejecución de sus obligaciones contractuales en más de un territorio nacional" (p.57), situaciones que, dado el avance tecnológico y la especialización de las profesiones, suelen tener lugar con mucha más frecuencia que antes. La Organización Internacional del Trabajo-OIT (OIT, 1988) ha recogido esta preocupación y desde 1919 busca promover y proteger el derecho de asociación tanto de trabajadores como empleadores (OIT, 1994) porque es a través de la fuerza concertada que ambas partes lograrán un desarrollo exitoso para el mejor cumplimiento de sus objetivos. Su función específica es promover la aplicación efectiva de los principios generales que rigen la libertad sindical. (OIT, 2006).

Los convenios más importantes y que enmarcan la legislación nacional son los Convenios 87, sobre libertad sindical y protección del derecho de sindicación, y el 98, sobre el derecho de sindicación y de negociación colectiva (OIT, 2006); especial mención también merece el Convenio 151 sobre las Relaciones de Trabajo en la Administración Pública, ello por la trascendencia de su efectiva aplicación al ámbito estatal. Asimismo, el Comité de Libertad Sindical ha emitido opinión sobre diversos aspectos, aquí una muestra: 
Sobre inculpación y condena de sindicalistas: "El Comité señaló el peligro que para el libre ejercicio de los derechos sindicales representan las condenas (...) relacionadas con la defensa de los intereses de sus mandantes" (OIT, 2006 p.24).

Sobre derechos de reunión y manifestación: "El derecho de las organizaciones (...) a celebrar reuniones (...) para examinar cuestiones profesionales (...) constituye un elemento fundamental de la libertad de asociación (...)". (OIT, 2006 p.31).

Sobre libertad de expresión: “(...) trabajadores como los empleadores deberían disfrutar de libertad de opinión (...)”. (OIT, 2006 p.35).

Sobre autorización y censura de publicaciones: “(...) la imposición de la censura de prensa durante un conflicto profesional puede tener un efecto directo sobre la evolución del conflicto y perjudicar a las partes (...)". (OIT, 2006 p.38).

\section{Denuncias Sindicales ante la OIT.}

a) Queja contra el Gobierno de Perú, presentada por la Confederación de Trabajadores del Perú (CTP) y la Federación Nacional de Trabajadores del Programa Nacional de Apoyo Directo a los Más Pobres "Juntos" (FENATRAJUNTOS).(...) (OIT, 2015, pp 714-722).

b) Queja contra el Gobierno del Perú, presentada por la Central Unitaria de Trabajadores del Perú (CUTPerú) apoyada por la Confederación Sindical Internacional (CSI). (...) (OIT, 2015, pp 762-768).

c) Queja contra el Gobierno del Perú, presentada por el Sindicato Nacional Centro Unión de Trabajadores del Seguro social de Salud (SINACUT-ESSALUD) y la Confederación General de Trabajadores del Perú (CGTP).

d) Queja contra el Gobierno del Perú, presentada por la Confederación General de Trabajadores del Perú (CGTP) y el Sindicato de Obreros Mineros de Shougang Hierro Perú y Anexos (SOMSHYA). (OIT, 2015, pp 826-832).

e) Queja contra el Gobierno del Perú, presentada por el Sindicato de Trabajadores Funcionarios de la Compañía Minera ANTAPACCAY (SITRAMINA).(...)". (OIT, 2015, p 854).

\section{DISCUSIÓN}

La Falta de regulación tuitiva y el inadecuado ejercicio del derecho colectivo del trabajo, aplicada en el momento actual, muestran una correlación directa con la variable dependiente Ineficacia de la Ley de Relaciones Colectivas de Trabajo en la Región Tacna-2015. También se ha determinado una disminución de las organizaciones sindicales del sector privado en contraposición con un incremento de las organizaciones del sector público, prácticamente ha disminuido considerablemente el trabajo asalariado, principal espacio donde las relaciones colectivas han tenido su origen y desarrollo (Córdova, 1981), dando cabida a nuevas formas laborales (autoempleo, subempleo, trabajo independiente), lo que dificulta el fortalecimiento de las relaciones colectivas. El aparente surgimiento de nuevos sindicatos, sobre todo en el sector público, es indicativo también de un debilitamiento de las relaciones colectivas, porque en su mayoría este crecimiento responde a la división de grupos sindicales en secciones más pequeñas. Asimismo, se ha determinado un debilitamiento de la habilidad o interés de las partes para negociar en trato directo ya que, en la mayoría de los casos que se presentan en su quehacer empresarial y que, sin pertenecer al procedimiento regular de una negociación colectiva (pliego de reclamos), causen tensión en las relaciones laborales, prefieren resolverlo con la intervención estatal a través de reuniones extra proceso, lo que aunque resulte beneficioso para evitar situaciones de conflicto peores, no resulta una medida idónea para la promoción del respeto a la autonomía colectiva que propugna la OIT, sino que ayuda más bien al debilitamiento de las relaciones colectivas, tanto de trabajadores como de los mismos empresarios.

Con el análisis de la situación en la región de Tacna se aprecia que los sindicatos no tienen una posibilidad real de nivelar el poder económico que tienen las empresas, ya que su fuerza radicaba en la unidad; con el 
fraccionamiento de organizaciones sindicales se tiene también un fraccionamiento de los intereses de grupo. Lo que inviabiliza cualquier negociación.

Asimismo, las normas vigentes en materia de negociaciones colectivas de trabajo no han contemplado la circunstancia de inexistencia o disminución del sector laboral productivo, por lo que medidas como la tercerización fácilmente restan capacidad negociadora a los trabajadores.

En cuanto al factor o factores que inciden en el debilitamiento de las organizaciones sindicales, tenemos que el decrecimiento de las actividades sindicales de los gremios en las dos últimas décadas, sea porque la actividad empresarial formal también ha decrecido habiendo desaparecido muchos centros de trabajo. Asimismo, debe considerarse también la indiferencia que se observa en relación a los reclamos efectuados por los servidores públicos que aún no ha merecido atención por parte del Estado. Por otro lado, considerando que con la dación de la Ley № 27912, la cancelación de registro sindical no se tramita administrativamente si no media mandato judicial, a la fecha se tiene un gran número de sindicatos registrados pero que no ejercen una vida sindical activa, inclusive muchos de ellos se han disuelto de hecho. La Ley de Relaciones Colectivas de Trabajo, así como su reglamento y modificatorias, estuvo pensada para un momento histórico en el que nuestro país tenía una realidad empresarial económicamente fuerte y concentrada en la zona urbana. Desde entonces han pasado más de veinte años, por lo que es obvio que la realidad económica también ha variado.

Se propone la modificación del Texto Único Ordenado de la Ley de Relaciones Colectivas de Trabajo, Decreto Supremo 010-2003-TR, que debería efectuar el Gobierno Central; no solo para cumplir las recomendaciones de la OIT, sino para incluir al sector laboral del régimen MYPE,

\section{REFERENCIAS BIBLIOGRÁFICAS}

Ackerman, M., Albuquerque, R, De Buen, N, Pasco, M., Giglio, W., Montoya, A., Plá Rodríquez, A. (1996). La Huelga en Iberoamérica. Lima: AELE.

Arévalo, J. (2005). Derecho Colectivo del Trabajo. Lima: GRIJLEY

Banco Central de Reserva del Perú. Caracterización del Departamento de Tacna.- Sucursal Arequipa (2015).

Carnelutti, F. (2008). Cómo Nace el Derecho. Bogotá: TEMIS.

Compendio Estadístico Perú 2014. Recuperado de

Córdova, E. (1981). Las Relaciones colectivas de Trabajo en América Latina. OIT. Ginebra, Suiza.

Dirección General de Juegos de Casino y Máquinas Tragamonedas-DGJCMT, Ministerio de Comercio Exterior y Turismo (2016). Distribución Geográfica de las Salas de Casino y Máquinas Tragamonedas. Recuperado de

Engels, F. y Marx, C. (2006). Ludwig Feuerbach y el Fin de la Filosofía Clásica Alemana. España

García, D. (1996). La Constitución en el Péndulo. Arequipa. Editorial UNAS Instituto Peruano de Economía (2014). Jiménez, R. (2009). Historia del Trabajo y del Movimiento Sindical. México. Colección

Escuela de Formación Sindical. Ediciones EEDUT

Kant, I. (1928). Crítica de la Razón Pura. Edición Digital. Recuperado de Kunz, A. y Cardinaux, N. (2005). Investigar en Derecho. Buenos Aires: Ed. Facultad de Derecho UBA.

Martínez Muñoz, J.A. (2007). La Autodeterminación, Anuario de Derechos Humanos, 08, pp 325-326. Recuperado de Ministerio de Trabajo y Promoción del Empleo.

Ministerio de Trabajo y Promoción del Empleo (Abr-Jun 2013). Conflictos Laborales. Reporte Trimestral. Neves, J. (2000). Jurisprudencia en materia laboral. Cuadernos de Debate Judicial (5). Lima: PUCP.

Neves Mujica, J., Herrera Vásquez, R., Cornejo Vargas, C., Pasco Cosmópolis, M., Martín Valverde, A., Dolorier Torres, J., Saco Barrios, R.G. (2010). Derecho Colectivo del Trabajo. Lima: Jurista Editores E.I.R.L. 
Oficina General de Estadística y Tecnologías de la información y Comunicaciones (2011). Las Huelgas en Perú 2011.Ministerio de Trabajo y Promoción del Empleo.

Oficina Internacional del Trabajo (12-27, marzo, 2015). 374은 Informe del Comité de Libertad Sindical. GB.323/INS/9. Ginebra, Suiza.

Ojeda, J., Tello, R. y Hernández, B. (2006). Análisis y Propuesta para el fortalecimiento de cadenas productivas en las zonas de influencia directa de Southern Perú. Notas Científicas. 9(2) 76-77. Recuperado de

Organización Internacional del Trabajo (1988). La Libertad Sindical-Manual de Educación Obrera. Ginebra. Suiza.

Organización Internacional del Trabajo (1994). Libertad Sindical y Negociación Colectiva. Ginebra. Suiza.

Organización Internacional del Trabajo (1986). Las Negociaciones Colectivas -Manual de Educación Obrera. Ginebra. Suiza.

Organización Internacional del Trabajo. Libre Funcionamiento de las Organizaciones Sindicales. Consejo Sindical Unitario de América Central y Caribe. Recuperado de Organización Internacional del Trabajo (2006). La Libertad Sindical. Recopilación de decisiones y principios del Comité de Libertad Sindical del Consejo de Administración de la OIT. Ginebra. Suiza.

Organización Internacional del Trabajo (2015). Protección de los Trabajadores en un Mundo del Trabajo en Transformación. Ginebra. Suiza.

Ormachea, I. (2000). Manual de Conciliación Procesal y Pre Procesal. Revista (3) Edición Especial.

Pasco, M. (1994). El Principio Protector en el Derecho Procesal del Trabajo. Revista de Derecho 48/1994. PUCP. Recuperado de

Picón, O. (2013), El potencial de la Zona Franca de Tacna. Conexión ESAN. Recuperado de

Rendón, J. (1998). Derecho del Trabajo Colectivo. Lima: EDIAL.

Sanguinetti, W. (2013). Derecho del Trabajo, Tendencias Contemporáneas. Lima: GRIJLEY.

Sindicato Unitario de Trabajadores de Telefónica del Perú S.A. v. FETRATEL. Exp. 1124-2001-AA/TC. (2002).

Sindicato de Trabajadores de la Municipalidad de San Borja. v. Municipalidad de San Borja. Exp. 13102011-PA/TC. (2011)

Toyama, J. (2015). Los grandes errores en las relaciones laborales ¿Se pueden evitar?. Miranda \&Amado Abogados. 